\title{
Receiver Operating Characteristic Curve of Total Serum IgE
}

\author{
in Allergic Patients
}

\author{
Haitham Bader Fathi *
}

\begin{abstract}
Background and objective: Validity of continuous variable test like total serum IgE is performed by constructing Receiver Operating Characteristic (ROC) curve. ROC curve is a technique for visualizing, organizing and selecting classifiers based on their performance. The study aims to determine the performance and validity of total serum IgE in various allergic diseases, age groups and gender. To select the optimal cut off value that has the best discriminative capability. To assess the sensitivity, specificity, predictive value and likelihood ratio of the selected cut-off points.

Materials and method: A diagnostic Receiver Operator Characteristic ROC curvestudy was conducted at Allergy Clinic, Al-Jamhouri Teaching Hospital in Mosul, Iraq on 751 individuals (561 patients with various allergic disorders and 190 healthy non-allergic subjects) ranged in age from 10 to 59 years. Classification of study samples into allergic or healthy groups was done by a committee panel of two qualified and expert allergiologist. A blood sample was taken for estimating total serum IgE by ELISA.
\end{abstract}

Results: The constructed ROC curve of overall sample showed that total serum IgE has moderate accuracy in allergic diseases and the AUC \pm SE was $0.730 \pm 0.022$ with $95 \%$ confidence interval $(0.686$ 0.773). Urticaria ROC curve had the maximum AUC $(0.742 \pm 0.030)$ and asthma had the minimum AUC $(0.720 \pm 0.027)$. The maximum AUC was detected in the age group 10-19 years and the least one was in age 50-59 years. No difference was found in the estimated AUC in both gender. The optimal cut-off point of total serum IgE was $96 \mathrm{IU} / \mathrm{ml}$ in the overall allergic patients and varies between $95 \mathrm{IU} / \mathrm{ml}$ (in urticaria) and $115 \mathrm{IU} / \mathrm{ml}$ (in rhinitis). Assessment of selected overall cutoff point of total serum IgE reveals the following: sensitivity (79.3\%), specificity (61.0\%), validity (74.7\%), PPV (50.0\%), NPV (85.7\%), LH+ (2.03), LH-(0.34).

Conclusion: Total serum IgE has moderate accuracy in diagnosis of various allergic diseases. Its accuracy is much better in younger patients in comparison to elderly. A cut offpoint of total serum $\operatorname{IgE}(100 \pm 5 \mathrm{IU} / \mathrm{ml})$ was the best classifier. Total $\mathrm{IgE}$ is a useful screening rather than a confirmatory test.

Key word: Total IgE, Allergy, ROC curve

\section{INTRODUCTION}

Diagnostic tests play a vital role in modern medicine not only for confirming the presence of disease but also to rule out the disease in individual patient. The validity of a test compared with the gold standard is determined by sensitivity and specificity. These two are components that measure the inherent validity of dichotomous test (Zhou et. al, 2002). Validity of continuous variable (polytomous) test (test that yield continuous numerical values like total serum IgE) is performed by constructing Receiver Operating Characteristic (ROC) curve (Zweig and Campbell 1993). This curve is technique for visualizing, organizing and selecting classifiers based on their performance (Sing et. al, 2005). It has long been used in signal detection theory to depict the trade of between hit rates and false alarm rates of classifiers. ROC analysis has been extended for use in visualizing and analyzing the behavior of diagnostic systems and in decision making (Kumar and Indrayan, 2011). Atopy is a genetic state in which exuberant production of $\operatorname{IgE}$ occurs as response to common environmental allergen (Owen, 2007). Almost $20 \%$ of people worldwide are considered as atopic individuals (Jarvis and Burney,1998) and their prevalence is increasing and nowadays described as epidemic (Isolauri et. $a l, 2004)$. Despite thehuge size of the problem, their diagnosis is usually based on clinical criteria due to unavailability of gold standard diagnostic instrument till now.Unfortunately, clinical criteria are subjective with low inter-observer agreement.

Allergic diseasesare usually associated with high total serum IgE level compared to healthy individuals (Baldacci et. al, 2001). However, it is unclear how total $\operatorname{IgE}$ measurements should be interpreted and to what extent total $\mathrm{IgE}$ measurements may contribute to decision-making in clinical practice. Inaddition, what is the minimum cut off value that can be used as best classifier. This knowledge gap prompt us to conduct this study aiming to construct ROC curve of total IgEand evaluate

* Dept. of Medicine, Ninevah College of Medicinel University of Mosul 
their performance in various allergic disease. Also, to select the optimal cut off value in various allergic diseases, age groups, and both genders.

\section{MATERIALS AND METHOD}

The sample of patients of this study had been recruited from the attendants of the Allergy Clinic at Al-JamhouriTeaching Hospital in Mosul/Iraq from April 2012 to Oct. 2012. All eligible patients who fulfill the inclusion criteria (history and clinical examination of one of the atopic diseases, aged between 10-60 years, and currently not using immunosuppressive medications) were asked to participate in the current investigation. Confirmation of the diagnosis of allergy was done by a committee of two allergiologist both are holder of higher Diploma in allergy and have more than 10 years clinical experience.

One hundred ninety healthy individuals were randomly selected fromthe attendance of the outpatient clinic. The selection criteria were based on absence of personal or family history of atopic diseases, normal eosinophil count, no history of worm infestation and in the age range 10 to 60 years.

Five $\mathrm{ml}$ blood was taken from each participants for determination of total serum $\operatorname{IgE}$ using the DRG® IgE (EIA-1788) (DRG International Inc, USA) by ELISA. Venous blood was centrifuged and serum was stored till testing.

\section{Assessment of test performance:}

To show the discriminative capability of total $\operatorname{IgE}$ between those with allergy and without, ROC curve was constructed by graphically displaying of sensitivity (true positive rate) on yaxis and (1 - specificity) False positive rate on $x-$ axis for varying cut-off points of test values (Greiner etal, 2000).

\section{Measuringvalidity of test:}

This was done by calculating total area under the curve (AUC) \pm standard error and $95 \%$ confidence interval by the use of non parametric Mann Whitney U test. The Area Under the Curve (AUC)is expect to have a value between 0.5 (chance) and 1 (perfect test). The larger the AUC the better test discriminative capability and validity.

\section{Selecting cut off value:}

Two methods were usedin selecting the optimal threshold point that gives maximum correct classification (these areYouden index and the point closest to " $0,1 ")$.

Youden index $\boldsymbol{J}$ is the point on the ROC curve which is farthest from line of equality (reference diagonal line) by the use of following equation (Fluss etal, 2005):

$\mathrm{J}=\max \left[\mathrm{S}_{\mathrm{N}}+\mathrm{S}_{\mathrm{P}}\right]$.

point closest to $(0,1)$ defined as the point on the ROC curve that has the least distance from upper left-hand corner estimated by the use of the following equation:

$\mathrm{d}^{2}=\left[\left(1-\mathrm{S}_{\mathrm{N}}\right)^{2}+\left(1-\mathrm{S}_{\mathrm{p}}\right)^{2}\right]$

The selected cut off point was the point that has the maximum Yoden index $\mathrm{J}$ and the least distance from $(0,1)$.

\section{Assessment of selected cut of value:}

The following definitions were used to assess assay performance (FN is a false-negative diagnostic test result, FP is a false-positive diagnostic test result, $\mathrm{TP}$ is a true-positive diagnostic test result, and TN is a true-negative diagnostic test result). After selecting cut of value for each disease condition and overall sample, following measures were used to assess their discriminative capabilities: sensitivity $\left(\mathrm{S}_{\mathrm{n}}\right)$ or true positive rate $[\mathrm{TP} /(\mathrm{TP}+\mathrm{FN}) \times 100]$ was defined as the percentage of positive test in allergic patients.Specificityor true negative $\operatorname{rate}\left(\mathrm{S}_{\mathrm{p}}\right)$ $[\mathrm{TN} /(\mathrm{FP}+\mathrm{TN}) \times 100]$ was defined as percentage of negative test in healthy subjects.Positive predictive value $[\mathrm{TP} /(\mathrm{TP}+\mathrm{FP}) \times 100]$ described the percentage of positive test that have allergic condition. Negative predictive value (PPV) $[\mathrm{TN} /(\mathrm{TN}+\mathrm{FN}) \times 100]$ is described as percentage of negative test that are healthy subjects. Efficiency $[(\mathrm{TP}+\mathrm{TN}) /(\mathrm{TP}+\mathrm{FP}+\mathrm{TN}+\mathrm{FN}) \times 100]$ describe the percentage of sample that are correctly classified by the test as having or not having allergy. The positive likelihood ratio is defined as true positive rate (i.e. sensitivity)/false positive rate (i.e. 1 - specificity). The negative likelihood ratio is defined as false negative rate (i.e.1 sensitivity)/true negative rate (i.e. specificity).

The construction and assessment of ROC curve was done by the use of statistical software SPSS ver 11 (Chicago Inc, ILL).

\section{RESULTS}

The studied sample consisted of 751 individuals. Their age ranged from 11 to 59 years with mean and standard deviation of 32.42 and 12.50 years respectively. They consist of $387(51.5 \%)$ males and $364(48.5 \%)$ females. The sample consist of 202(26.9\%) patients with allergic rhinitis, $176(23.4 \%)$ patients with bronchial asthma, 98(13.0\%) patients with dermatitis, $85(11.3 \%)$ patients with urticaria, and $190(25.3 \%)$ healthy non-atopic subjects. The details of the studied sample is shown in table 1 . 
ROC curve of overall studied sample is depicted in fig 1 . The estimated AUC \pm SE was $0.730 \pm 0.022$ with $95 \%$ confidence interval (0.686-0.773). The displayed curve reveals that the discriminative capability of total serum $\operatorname{IgE}$ lies about midway between useless test (AUC $=0.5$ ) which represented by reference diagonal interrupted line and ideal test $(\mathrm{AUC}=1)$ which reach the upper left-hand corner.

The ROC curve of individual allergic disease is depicted in fig 2. Estimation of AUC revealed that maximal area found in urticaria
$0.742 \pm 0.030 \quad(95 \% \mathrm{CI}=0.681-0.801)$ and the minimal in asthma $0.720 \pm 0.027(95 \% \mathrm{CI}=0.668$ 0.772). The estimate AUC in dermatitis was $0.742 \pm 0.030 \quad(95 \% \quad \mathrm{CI}=0.681-0.801)$ and in allergic rhinitis was $0.721 \pm 0.026 \quad(95 \%$ $\mathrm{CI}=0.678-0.780$ ).

The estimated AUC of total serum IgE in different age groups and both genders is shown in table 2. The maximum AUC was detected in the age group 10-19 years and the least one was in age 50-59 years. The AUC of males was slightly higher than females.

Table (1): The age and gender distribution of the studied sample

\begin{tabular}{|l|c|c|c|c|}
\hline \multicolumn{1}{|c|}{ Group } & No. & Mean age \pm SD & \% Males & \% Female \\
\hline \hline Rhinitis & 202 & $32.7 \pm 12.3$ & $55.9 \%$ & $44.1 \%$ \\
\hline Asthma & 176 & $32.9 \pm 13.6$ & $52.3 \%$ & $47.7 \%$ \\
\hline Dermatitis & 98 & $33.0 \pm 11.2$ & $50.0 \%$ & $50.0 \%$ \\
\hline Urticaria & 85 & $31.8 \pm 12.6$ & $47.1 \%$ & $52.9 \%$ \\
\hline Healthy & 190 & $29.8 \pm 11.5$ & $48.9 \%$ & $51.1 \%$ \\
\hline Overall sample & 751 & $32.42 \pm 12.50$ & $51.5 \%$ & $48.5 \%$ \\
\hline
\end{tabular}

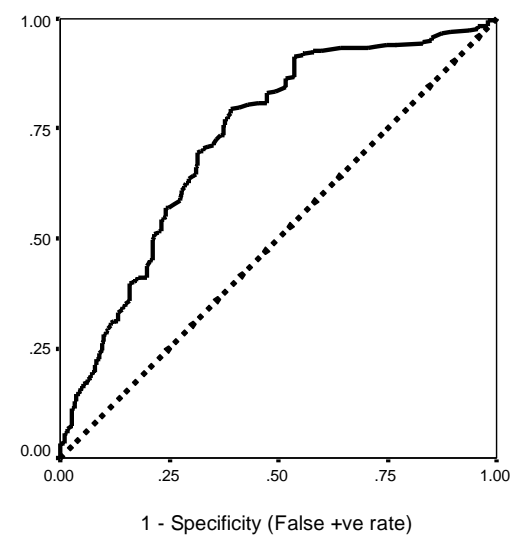

Fig. (1): ROC curve of total serum IgE in whole studied sample.

$(-)$ represent $\operatorname{IgE},\left({ }^{\cdots}\right)$ is the reference line.

(a)

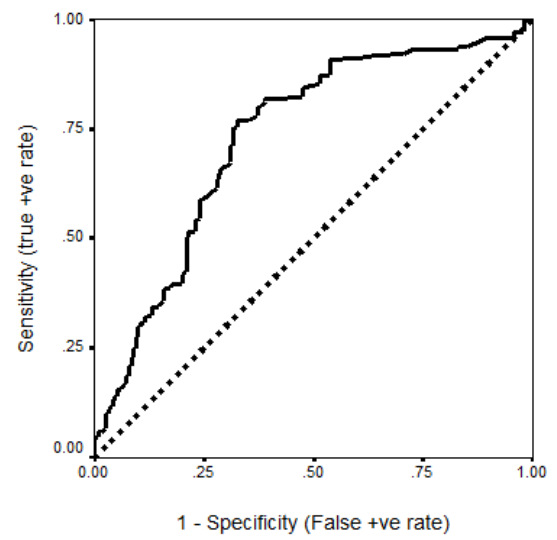

(b)

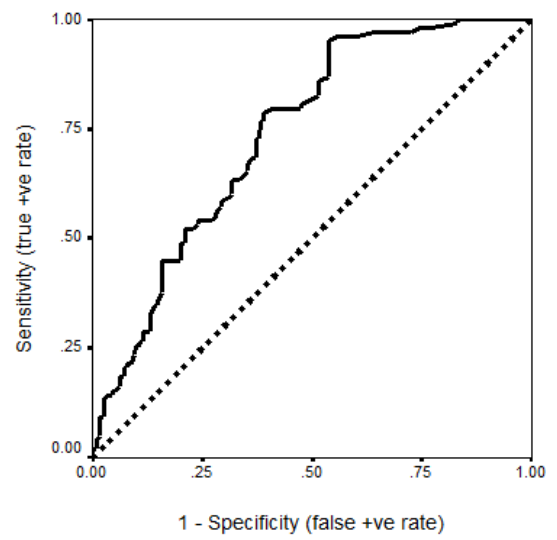


(c)

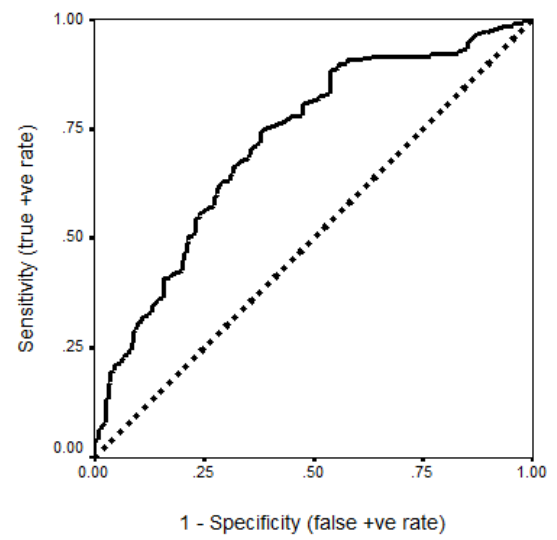

(d)

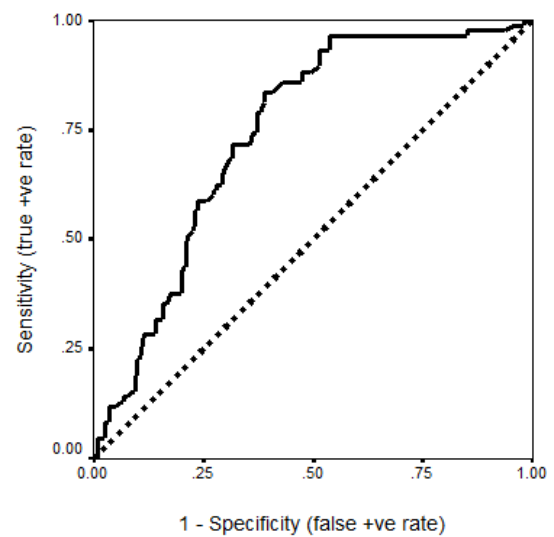

Fig. (2): ROC curve of total serum IgE in various allergic disease. Allergic rhinitis (a), bronchial asthma (b), dermatitis (c), urticaria (d).

$(-)$ represent $\operatorname{IgE},(\cdots)$ is the reference line.

Table (2): The estimated Area Under Curve (AUC) of total serum IgE according to age groups and gender

\begin{tabular}{|c|c|c|c|c|}
\hline \multirow{2}{*}{ Variables } & \multicolumn{2}{|c|}{ No. of individuals } & \multirow{2}{*}{ Mean $\mathrm{AUC} \pm \mathrm{SE}$} & \multirow{2}{*}{$95 \% \mathrm{CI}$} \\
\hline & Allergic & Healthy & & \\
\hline \multicolumn{5}{|l|}{ Age groups } \\
\hline $10-19$ years & 112 & 48 & $0.809 \pm 0.039$ & $0.733-0.886$ \\
\hline $20-29$ years & 148 & 68 & $0.735 \pm 0.038$ & $0.661-0.810$ \\
\hline $30-39$ years & 133 & 36 & $0.679 \pm 0.058$ & $0.564-0.793$ \\
\hline $40-49$ years & 103 & 23 & $0.707 \pm 0.002$ & $0.600-0.813$ \\
\hline 50-59 years & 65 & 15 & $0.661 \pm 0.074$ & $0.516-0.806$ \\
\hline \multicolumn{5}{|l|}{ Gender } \\
\hline Males & 294 & 93 & $0.732 \pm 0.031$ & $0.671-0.793$ \\
\hline Females & 267 & 97 & $0.722 \pm 0.032$ & $0.660-0.784$ \\
\hline
\end{tabular}

Table (3): The cut off value of total IgE that has the maximal discriminative threshold of overall studied sample and individual allergic disease with their performance

\begin{tabular}{|l|c|c|c|c|c|c|c|c|c|c||}
\hline $\begin{array}{l}\text { Type of } \\
\text { allergy }\end{array}$ & IgE & Sn & Sp & PPV & NPV & Validity & JI & $\begin{array}{c}\text { Point closest } \\
\text { to (0,1) }\end{array}$ & LR+ & LR- \\
\hline \hline Rhinitis & 115 & 76.2 & 67.4 & 71.3 & 72.3 & 72.0 & 1.44 & 0.16 & & \\
\hline Asthma & 102 & 73.9 & 62.1 & 64.4 & 72.0 & 67.7 & 1.36 & 0.21 & & \\
\hline Dermatitis & 98 & 76.5 & 61.6 & 50.7 & 83.6 & 66.6 & 1.38 & 0.20 & & \\
\hline Urticaria & 95 & 83.5 & 61.1 & 49.0 & 89.2 & 68.0 & 1.45 & 0.18 & & \\
\hline Overall & 96 & 79.3 & 61.0 & 50.0 & 85.7 & 74.7 & 1.4 & 0.20 & 2.03 & 0.34 \\
\hline
\end{tabular}

IgE (cut off value of total serum IgE), Sn (sensitivity), Sp (specificity), PPV (positive predictive value), NPV (negative predictive value), JI (Youden index), LR+ (positive likelihood ratio), LR- (negative likelihood ratio)

\section{DISCUSSION}

Assessment of allergic status by measuring the serum immunoglobulin ( $\mathrm{Ig}$ ) $\mathrm{E}$ antibodies is fast and safe procedure. Serum antibodies can be assessed in patients with dermatitis even if they use antihistamines or other pharmacological agents that reduce skin sensitivity (Baldacci et. al, 2001).

To the best of our knowledge, this is the first study of its kind conducted in our locality 
attempting to re-evaluate the usefulness of total serum IgE as a marker of allergic diseases on a large sample (751) of allergic patients and healthy subjects.

The results of diagnostic studies are threaten by large number of biases (like, gold standard bias, verification bias, selection bias, test review bias, and un-interpretable test result) that may spoil the result, making their generalization difficult (Whiting et. al, 2004). In the current study we used many strategies to manage and minimize the well known biases (Clark, 2008). Lack of gold standard diagnostic assay was solved by the use "opinion of panel of experts rather than single investigator" in order to improve the validity of clinical criteria and reduce the effect of gold standard bias. Equal chance in interviews and investigations were provided to both patients and healthy non-allergic subjects in order to minimize the verification bias. Because test may perform differently in different disease severity, full spectrum of condition was selected to decrease selection bias. The estimation of total serum $\operatorname{IgE}$ is fully automated and done by a lab technician totally blind to the diagnosis of the participants. These help in removing the threat of inter-observer and test review biases. None of the yielded results were omitted in order to reduce the effect of uniterpretable result.

The underlying assumption of ROC analysis is that a diagnostic variable (like ELISA total IgEvalues) is used to discriminate between two mutually exclusive states of tested individuals (like allergic and non-allergic individuals).The area under the ROC curve is a global summary statistic of diagnostic accuracy (Hanley and McNeil, 1992). .A proposed guideline for assessing test accuracy (based on yielded AUC) is as follows: (AUC $=0.5$ ) noninformative or useless test, (AUC >0.5-0.7) less accurate test, (AUC >0.7-0.9) moderately accurate test, (AUC >0.9-1) highly accurate testand $(\mathrm{AUC}=1)$ as a perfect test (Hanley and McNeil, 1992). Accordingly, total serum IgE in the current study is considered as a moderately accurate test of allergic status. The estimated AUC in the current study is in agreement with the reported (AUC $=0.736$ ) by Kovacetal in their study on 157 children with allergic asthma (Kovac et. al, 2007). The results of this study show a gradual decrease of AUC with age. A similar observation was reported by other researchers and concluded that discriminative capability and performance of total serum $\operatorname{IgE}$ is much better in children in comparison to their performance in elderly.

Choosing the optimal decision is a tradeoff between optimizing sensitivity and specificity (Obuchowski et. al, 2004). The optimal decision thresholds obtained in this analysis were selected assuming the cost of false-positive result and false-negative result were equal, but this may not be the optimal in all clinical applications. The optimal decision threshold for specific application involve a number of factors that are not the properties of the testing system, rather they are the properties of clinical application. These include prevalence, the cost of misclassification and the outcome. All these factors interact with the result of the test affectingtheir usefulness in clinical practice.

The optimal cut offpoint (i.e, point that has max Youden index and closest to "0,1" corner) of total serum IgE in the current study ranged from $95 \mathrm{IU} / \mathrm{ml}$ (urticaria) to $115 \mathrm{IU} / \mathrm{ml}$ (rhinitis). The yielded figure was lower than that recommended by the manufacturer(i.e, $150 \mathrm{IU} / \mathrm{ml}$ ), but it was in agreement with the figure reported by TENOR (Epidemiology and Natural History of Asthma: Outcomes and Treatment Regimens) which is large $(4,500)$ cohort of patients with severe and/or difficult-to-treat asthma ${ }^{8}$. The figure reported by TENOR was $103 \mathrm{IU} / \mathrm{ml}$. They also concluded that test performance was better in those $<18$ years in comparison to those $\geq 18$ years. The selected point shows a higher sensitivity on the cost of specificity. This indicates that total serum IgE is more useful as a screening rather than a confirmatory test.

\section{CONCLUSION}

In conclusion, total serum IgE has moderate accuracy in the diagnosis of various allergic diseases, its accuracy is much better in younger patients in comparison to elderly, and no gender difference was noticed in their performance. A cut of value of $100 \pm 5 \mathrm{IU} / \mathrm{ml}$ is the best classifier. Total IgE is a screening rather than confirmatory test.

\section{REFERENCES}

Baldacci, S.; Omenaas, E.; Oryszczyn, MP. (2001). Allergy markers in respiratory epidemiology. EuResp J. 17. P.p.773-90

Clark, RD.; Webester-Clark, DJ. (2008). Managing bias in ROC curves. $J$ ComputMol Des. 22. P.p.141-46

Fluss, R.; Farggi, D.; Reisser, B. (2005). Estimation of Yoden index and its associated cut of point. Biom $J$. 47.P.p. $458-72$ 
Greiner, M.; Pfeiffer, D.; Smith, RD. (2000). Principles and practical application of the receiver-operating characteristic analysis for diagnostic tests. Prev Vet Med. 45. P.p.23-41

Hanley, JA.; and McNeil, BJ. (1992). The Meaning and Use of the Area Under a Receiver Operating Characteristic (ROC) Curve. Radiology. 143. P.p. 29-36.

Isolauri, E.; Huurre, A.; Salminen, S.; Impivaara, O. (2004). The allergy epidemic extends beyond the past few decades. ClinExper Allergy. 34. P.p.1007-10

Jarvis, D.; Burney, P. (1998). The epidemiology of allergic diseases. BMJ. 316. P.p.607

Kovac, K.; Doding, S.; Tiesic-Drunkovic, D.; Roos, M. (2007). Correlation y and serum $\operatorname{IgE}$ in asthmatic children sensitized to DermatophagoidsPeteronyssinus. Arch Med Res. 38. P.p.99-105

Kumar R, Indrayan. (2011). A Receiver Operating Characteristic (ROC) Curve for Medical Researchers. Indian Ped. 48. P.p.277-67
Obuchowski, NA.; Lieber, ML.; Wians, FH. (2004). ROC Curves in Clinical Chemistry: Uses, Misuses, and Possible Solutions. ClinChem. 50. P.p.1118-25

Owen, CE. (2007). Immunoglobulin E: role in asthma and allergic disease: lessons from the clinic. PharmacolTher. 113. P.p.12133.

Sing, T.; Sander, O.; Beerenwinkl, N. (2005). ROC: visualizing, classifier, performance. Bioinformatics. 21 . P.p.3940-41

Whiting P, Ruljes AW, Reitsma JB, Glas AS, Bossuyt PM, Kleijnen J (2004). Sources of variation and bias in studies of diagnostic accuracy - a systematic review. Ann Intern Med.140. P.p.189202.

Zhou, XH.; Obuchowski, NA.; McClish, DK. (2002). Statistical methods in diagnostic medicine. ( $1^{\text {st }}$ ed.). New York: John Wiley \& Sons. P.p. 15-164

Zweig, MH.; Campbell, G. (1993). Receiver Operating Characteristic (ROC) Plots: A Fundamental Evaluation Tool in Clinical Medicine. ClinChem. 39. P.p.561-77. 